\title{
The Closing of IMA and MBI
}

\section{Nilima Nigam}

Editor's Note: The Division of Mathematical Sciences (DMS) of the National Science Foundation (NSF) has a program that supports eight major mathematics institutes in the United States. Included among these are the Institute for Mathematics and its Applications (IMA) at the University of Minnesota and the Mathematical Biosciences Institute (MBI) at Ohio State University. In January of this year, news circulated that the DMS planned to ramp down significantly its funding of IMA and MBI. This issue of the Notices features two articles on this topic. The first, an opinion piece by Nilima Nigam, professor of mathematics at Simon Fraser University, is below.

The second, a piece by DMS director Michael Vogelius and DMS senior advisor Henry Warchall that puts the matter into the larger context of a discussion of the DMS portfolio, follows on $\mathrm{p} 1375$.

Another unusual feature of the mathematical sciences is the mathematical sciences institutes. These are our community's large projects, our telescopes peering into the future and our microscopes focusing intently on the deepest fundamental problems, our laboratories experimenting with new configurations of people and ideas and a significant vehicle for cultural change in the mathematical sciences. The Committee of Visitors endorses the great value of the institutes, is happy with the way that they are managed, while recommending a few improvements, largely to adjust certain artifacts of the history of the program.... [3]

\section{Introduction}

Since the beginning of 2015, the international community of mathematical scientists has been trying to absorb the impact of two recommendations from the Division of Mathematical Sciences (DMS) at the National Science Foundation (NSF): a call to end funding to the Institute for Mathematics and its Applications (IMA) and a call to end funding to the Mathematical Biosciences Institute (MBI) unless half its budget is funded through a different directorate within the NSF. As I write this, these recommendations have not been made official by the NSF, and the foundation therefore is not allowed

Nilima Nigam is professor of mathematics at Simon Fraser University. Her email address is nigam@math. sfu.ca.

For permission to reprint this article, please contact: reprint-permission@ams.org.

DOI: http://dx.doi.org/10.1090/noti1302 to publicly comment on the recommendation. In this note I seek to explain why I think the MBI and IMA are successful in their central missions and worthy of our continued support. These are my own views and opinions.

At this juncture what is visible to the broader scientific community is the dismantling of a communal infrastructure of great importance, which includes vital training programs and an entire mode of scientific interaction aimed at promoting the reach of the mathematical sciences in other areas of scientific inquiry.

We recognize this is a time of unique challenges in terms of government funding of fundamental scientific and mathematical research and that the NSF has had to respond to severe budgetary pressures. These pressures are unfortunately present and evident in many countries. It is inevitable that the math institutes would also, in turn, be asked to share in the pain. Responsible decision making in an already strained fiscal environment may involve choices which are difficult but should strive to minimize the cumulative negative impact.

In my personal view the current DMS recommendations concerning the MBI and IMA give the appearance of difficult choices courageously made which unfortunately seem to discount the central and ongoing importance of these two institutes to many mathematical scientists at all stages of their careers. These institutes have played a profound role in identifying interdisciplinary applications of many mathematical subdisciplines and supporting research groups that work in them. We have gone from a period when the mathematical sciences were isolated to an era where the centrality of mathematics in interdisciplinary endeavors is widely 
acknowledged. As an example, the 2006-07 IMA annual program in algebraic geometry helped identify applications in the area and launch a vibrant new field. There is now a SIAM activity group in algebraic geometry with scores of researchers. As the Committee of Visitors to the DMS stated in their 2013 report, the institutes are a "significant vehicle for cultural change" within our discipline. It is therefore surprising to me that, by the end of 2014, two of the institutes were being recommended for defunding.

Like many others, I have been the beneficiary of the rich intellectual environment and mathematical generosity of the US-based mathematical sciences community, especially through the institute programs. I am deeply indebted to the National Science Foundation and the US-based mathematical community for their truly generous support of my academic training and of others like me. I do not know and cannot speculate what factors led to the NSF recommendations.

\section{The Beneficiaries}

\begin{abstract}
...the mathematical sciences have encountered a growth in the number of mathematicians, the number of results, and the number of publications. Unfortunately, much of this growth has been centrifugal....Many of the questions which have led to the development of whole fields, and consequently the connections of these fields with the rest of mathematics and with other sciences, have been lost in the teacher-to-student chain. The historical linkage between mathematics and the sciences, which had been of such great mutual benefit in the past, is in need of nurture. [2]
\end{abstract}

The First Annual Report of the IMA by Hans Weinberger [2] contains a truly well-articulated and prescient vision of the needs of mathematical scientists embarking on interdisciplinary work. The quote above remains pertinent today. The mathematical sciences are vital and ever-growing at a pace which renders it difficult for any individual to keep abreast of all major developments. In parallel, new developments in other disciplines lead to interesting mathematical questions, which first need to be properly formulated. The risk for missed connections and insights remains high.

Developing and nurturing connections between the mathematical sciences and other disciplines requires a sustained effort on many levels. We need to train young mathematicians. We need to have a forum where our community can meet scientists from other areas and identify problems where mathematics can play a central role. We need to provide opportunities for sustaining nascent research disciplines. We need to provide an inclusive and intellectually stimulating environment for scholars seeking to develop a new area of application of mathematics. At its best, such work requires considerable intellectual risk taking, but enriches the application area immeasurably. It will produce novel mathematical insights, which in turn should be a source of continual regeneration [2] in our discipline. Supporting such endeavors is, and should continue to be, a priority.

It is in this setting that both the IMA and the MBI play a central role as shared large infrastructure. The IMA was founded in 1982 to spawn new areas of mathematical research through contact with the physical, engineering, and social sciences. The MBI was founded in 2001 to foster the development of new mathematical areas motivated by questions in the biosciences. The IMA and MBI programs include training initiatives aimed at students (undergraduate and graduate) and post-doctoral fellows (PDFs). They host well-attended workshops on newer topics as well as thematic annual programs. Hundreds of mathematical scientists access their programs each year, many for the first time. For many, these institutes provide a valuable regional hub for long-term training and research initiatives. The mentoring and networking initiatives have helped many younger researchers from diverse backgrounds. Certainly these visitors and trainees benefit from these institutes.

What about the mathematical sciences as a whole? As I describe below, both institutes have been very successful in sparking new research on mathematical questions motivated by applications. Our discipline as a whole is richer for their presence. Equally, thanks to the programming at these institutes, the impacts and development of new mathematical ideas in the application areas have been tremendous.

While the principle of seeking funding from receptor disciplines is reasonable, in practice this neglects the push-and-pull nature of research in applied mathematics. As new interdisciplinary fields develop and begin to have impact in the receptor area, the research rightly gets pulled into that field, and the insights developed by mathematicians become part of the mainstream practice. Young biologists now are increasingly trained to use quantitative tools. The more common the adoption of these tools, the less urgent it seems that we need to support new mathematical sciences research in these areas. I have heard it said: "But why do we need to support mathematics in robotics? Isn't that field sorted out?" However, if there is not a counterbalancing pull from the mathematical sciences side, over time a problem emerges. New insights in the mathematical sciences are not adopted in the receptor field, and, conversely, mathematicians lose contact with the newest developments in the application area. This cycle seems rather inefficient. Efforts to sustain interdisciplinary connections are vital. Fields such as mathematical biology are 
very much aware of this danger. Supporting the core mission-to sustain mathematical sciences research which is motivated by applications and to maintain contact with the applications areas-is integral to the health of our discipline.

\section{The New Generation}

I come from an ecology background and there was [sic] many incredibly helpful hands-on session [sic] on mathematical models that can be applied to ecology. Learning such methods was extremely beneficial for the development of my PhD. In addition, I found the talks inspiring and I am considering a few of the speakers as potential post-docs advisors. -MBI participant When I was a graduate student, I was having difficulty finding a project to pursue, and I was having doubts about whether to continue with my graduate education. A research mentor sent me to the IMA to attend my first applied mathematics conference, a 2001 meeting on Ideal Data Representation. ...This visit ultimately led to my first paper. Indeed, I owe my career as a researcher to this trip to the IMA. -Joel Tropp, Caltech

The training of future generations of mathematical scientists - undergraduates, graduate, and postdoctoral fellows-is an integral part of the IMA and MBI. Attracting students to research in mathematics takes effort. Both institutes have novel and highly successful Research Experience for Undergraduates (REU) programs. Their public lecture series are an integral part of the intellectual culture in their communities. Both institutes also set a high priority for the participation of graduate students in various programs. The impact of visits to these institutes can be profound on a fledgling career. A quick look at the editorial boards of the major journals in applied, computational, and industrial mathematics, as well as mathematical biology, shows that alumni from the IMA and MBI PDF programs are represented there in impressive numbers.

The IMA postdoctoral program was established in 1982. Since then, 296 PDFs have spent formative years at the IMA, immersed in the annual programs. This is a hugely successful program, providing young researchers with novel opportunities, intellectual support, and protected time to pursue ambitious research projects at the very forefront of applied mathematics. The program prioritizes fresh PhDs, for whom the IMA experience provides excellent training and career-related mentoring. While receiving support for collaboration with the visitors at the myriad IMA workshops as well as with faculty across many departments at the University of Minnesota, the PDFs learn to interact with a broad range of scientists. Perhaps less visibly, the advantage of interacting with each other and forming lasting research networks is an invaluable benefit for the PDFs. Postdocs from the IMA go on to successful careers in academe and industry and include thirteen Sloan Research Fellows and six NSF CAREER Award recipients. Alumni of the program hold positions in top-notch research departments and in the R\&D (research and development) groups of major technology firms.

The pioneering IMA industrial PDF program was established in 1988 and has since supported sixty-five industrial PDFs. The PDFs spend half of their two-year IMA stint working on an industrial research project at the company site with a mentor from the industrial R\&D group. This enables them to work on long-term projects of interest to the company and learn vital industry-relevant skills. The IMA provides critical support at all stages-identifying companies, matching the PDFs with local (IMA) and industrial mentors, ensuring the research project is well defined, and helping with knowledge transfer and intellectual property issues. Specific and often time-sensitive issues arise with industrial collaborations, and the IMA has accumulated a wealth of experience in dealing with these. The program is open to international scholars and serves as an important mechanism for the US to attract the best talent.

The MBI has hosted eighty-one PDFs since its inception. Each PDF has two mentors: one from the biological sciences and one from mathematics. The duration of these PDFs is three years, which is the usual length of time needed to establish collaborations and embark on an independent research program in mathematical biology. Like the IMA, the MBI puts a high priority on nurturing first-time PDFs.

In both institutions, NSF funding supports part of the PDF program. At the IMA it helps support one year of the PDF's stay. The remaining time is supported through other sources, including other departments at the University of Minnesota and industrial partnerships. At the MBI, some PDFs are supported through NSF funding for the duration of their stay; others are co-sponsored with biosciences researchers. Between them these institutes represent a large number of available opportunities for first-time PDF researchers who are in a vulnerable phase of their careers. The cessation of NSF funding will have a crippling effect on these PDF programs.

What will be lost with the defunding of these institutes? It can be argued that PDFs can be absorbed into individual departments. What will be lost is the opportunities they have access to: annual programs, scientists from other disciplines, visitors, mentorships, industrial interactions, and each other. Individual supervisors can seek to recreate 
some, but surely not all, aspects of this milieu. Similarly, one can envisage each component-graduate workshops, REU programs, outreach activitiessurviving in numerous institutions. What is lost is the synergy and interplay between them. One can replant ten trees, but that does not regenerate an ecosystem.

\section{The Growth of Knowledge}

Each yearly program is devoted to studying the possible interactions of mathematics with a specific area of science. Scientists with common interests are invited to be in residence for sufficiently long periods of time to allow interest in the nontrivial problems which arise to grow and familiarity to develop... -First IMA Annual Report This paper, and some of our recent work on which it draws, has its origins in a remarkable IMA workshop on gait patterns and symmetry held in June 1998, which brought together biologists, engineers, and mathematicians. ...Workshop discussions in which we all took part also inspired the creation of RHex, a six-legged robot... [4]

The reach and influence of the mathematical sciences is vast. Many scientific and engineering disciplines use mathematical ideas and tools; in fact, it is possible that we as mathematicians have not even fully appreciated the extent of the "unreasonable effectiveness" of our own discipline. The crystallization of some of the most exciting and promising research areas emerges from the interaction and cross-fertilization of different disciplines with the mathematical sciences. This does not happen in a vacuum. One needs an environment hospitable to both the applicationarea experts as well as the mathematical scientists. Scientists and mathematicians need a forum to interact and develop a common vocabulary. In the early stages it is not even clear which subdisciplines of mathematics will be most helpful. One needs a few nucleation sites: scholars who have the opportunity to interact with a large number of experts in more specialized areas, so they can begin to aggregate existing knowledge and help generate new ideas.

The long-term programs of the IMA and MBI have played a vital role in interdisciplinary exploration involving mathematics. This model of workshops and tutorials addressing different aspects of the overarching theme allows for both flexibility and depth. A large number of visitors specializing in different aspects of the new area gather for workshops. A smaller number of PDFs and longterm visitors stay in residence, participate in all the activities, develop a shared vocabulary, and obtain a bird's-eye view of the field. This is vital: connections between different topics do not emerge unless someone is present to see them. It is particularly important that PDFs have a long-term affiliation with reduced teaching responsibility in this setting; they are embarking on research in newly emerging areas, where even the specification of a core mathematical question will take time. This particular feature of applications-driven research also distinguishes the IMA and MBI programs from those which bring together different mathematical communities. In the latter setting, a great deal of shared knowledge already exists.

As an example, the vibrant area of mathematical materials science has deep links to the IMA. In the 1990s this was a relatively new field; many important research directions were formulated and explored during the IMA annual programs and workshops in the 1990s. This is a field which is now both established and mature (the SIAM Activity Group on Mathematical Aspects of Materials Science (SIAG/MS) was established in 2008), but the influence of the IMA is clearly seen: most of the leaders in this area have been visitors or PDFs of the IMA.

As another example, in 1998 the IMA held a workshop on animal locomotion. As Holmes, Full, Koditsche, and Guckenheimer describe in their 2006 paper [4], workshop discussions led to an extensive collaboration, which included the creation of hexapod robots (a project funded for many years by DARPA) and to NSF-funded work on the neural and muscular control feedback loops that govern legged locomotion. This has been a productive interaction which continues to influence developments in the fields of neuromechanics, robotics, and sensorimotor control, as well as in the mathematical study of dynamical systems.

Surely thirty years ago no one would have predicted that tools from algebraic topology would become important for sensor networks, actuators, and robotics. The IMA has, over the years, hosted programs on networks. There have been workshops on dynamical systems and actuators. Perhaps it was at one of these events that researchers identified the potential for using local-to-global principles from algebraic topology? I do not know for sure. What is certain is the huge impact of the 20132014 IMA Theme Year on applications of algebraic topology. This program brought together a truly wide array of mathematicians and scientists and included a workshop on topological systems in communication and sensing. A research network of over two hundred mathematicians was created, and their activities continue to be supported by the IMA. I can claim to have personally benefitted: as a young graduate student I made a bet with a fellow student who asserted algebraic topology would never be "sullied by relevance in the real world" during his lifetime. He is still alive, and now I am owed US\$5. 
The biological and biomedical sciences include problems of staggering complexity and scope which require a truly multidisciplinary approach. For instance: What is the role of stochasticity in biological systems? Does it serve a necessary purpose? To understand these questions, the 2011-2012 MBI Emphasis Year on Stochastics in Biological Systems brought together probabilists, algebraic geometers, engineers, epidemiologists, systems biologists, and other biological scientists. Open questions of mutual interest were identified and carefully formulated. On the biomedical side, consider the study of cancer. Oncology is by no means a new field, and the central importance of a vast range of mathematical ideas is clear: from the study of changes in the genome to modelling the effects of ablation therapy. It is also known that cancers interact with their environment in a complex manner at many scales, ranging from the level of genes and proteins to the level of entire populations. There is a range of therapies which target specific interactions. Is there a mathematical framework which can account for these disparate phenomena? Can it be used to generate predictions about the efficacy of new treatments? These are truly big questions, which are being studied during the 2014-2015 MBI Emphasis Year in Cancer and Its Environment. The role of the MBI is central.

\section{The Relationships}

Macalester has partnered with the IMA in running an applied mathematics REU for the past six years. ...The program involved scientists, engineers and mathematicians of all stripes: university researchers, liberal arts professors, post-doctoral fellows, graduate and undergraduate students. Everyone got something out of participating in this focused research community, not just the undergraduates. This broad impact was by design, and credit for that revolutionary approach goes to the IMA.

- Andrew Beveridge, Macalaster

While a graduate student, I received funding to travel to the IMA and participate in the workshop "Fostering Mathematical Entrepreneurship." Even though I felt confident I would be able to secure a post doc position when I finished my PhD, I was interested in work outside academia, and so naturally the above IMA program was very appealing....The workshop was a hugely important experience for me, a pivotal part of my graduate and professional career.

-Zachary Gelbaum, Spacetime Capital

Management \& Research, LLC

Consider first the numbers. The IMA hosts around 1,200 visitors each year, of which 50 percent are first-time visitors. These numbers include graduate students (24 percent) and nonmathematicians (32 percent). Nearly 50 percent of the visitors apply themselves (i.e., are not selected by the organizers). MBI, the younger (and smaller) of the two institutes, has hosted over 9,200 visitors since 2002. If you had to design a method for efficiently mixing a population of mathematicians and scientists, ensuring serendipitous interactions, you would be hard pressed to find a more successful model than these institutes.

The role of the IMA and the MBI in creating new research communities at the interface of mathematics and other disciplines has already been discussed. Relationships with R\&D groups in industry are, if anything, even more challenging to initiate. Almost surely, mathematicians seeking to build links with companies on their own will find the process time consuming and frustrating. The IMA has dedicated significant efforts to nurturing these activities and relationships through a range of programs and seminars. The graduate student problem-solving workshops have already been mentioned, as has the industrial PDF program. Colleagues from the eleven IMA corporate partners provide mentorship and valuable career advice to young mathematicians at the IMA. This model of fostering relationships between industry and the academy through PDFs has proven very successful, and the IMA model has inspired similar initiatives. The NSF GOALI (Grant Opportunities for Academic Liaison with Industry) program comes to mind, as does the MITACS industrial internship program in Canada (a national research organization that manages and funds research and training programs for undergraduate, graduate students, and postdoctoral fellows in partnership with universities, industry, and government in Canada). The learning curve for any individual researcher attempting such activities is high, and the barriers to success are formidable. I spent time at MITACS as these programs were being developed and often looked to the IMA programs for guidance. The IMA is the place I recommend that students visit if they are contemplating careers in industry: they will receive excellent mentoring, ideas of career paths, opportunities to network with mathematicians in companies. The suite of IMA industrial programs is unique in North America in terms of scope and active involvement by industrial partners.

Individuals can seek to recreate these relationships, but it is hard to achieve the same scale. The loss of both institutes in the Midwest will have a critical effect on many colleagues: for many students, the geographical proximity of these institutes is an important factor in their ability to attend conferences. Interactions and collaborations made possible through the IMA and MBI will now have yet another barrier. The DMS will have essentially dismantled the only sustained forum for the training of industrial PDFs in the mathematical sciences. 


\section{The International Influence}

In the crucial field of understanding the basics of biology in strict (i.e. mathematical) terms the US is so much in advance of us (the rest). This will lead not only to fundamental breakthroughs, comparable to those of physics a century ago but also to new medical technologies. To a great extent this seems to be the consequence of a conscious and concerted effort from the leadership of the National Science Foundation. Wish we could have something similar in Europe.

-MBI participant

Of the many modes of scholarly research supported by the NSF, a highly efficient mechanism in terms of international impact has been through the institutes. The leadership role and importance of the IMA and the MBI cannot be emphasized enough. Globally, we collectively learn and benefit from their expertise on how to enhance links between the mathematical sciences and applications.

As a concrete example, in 2008 the International Commission on Mathematical Instruction (ICMI) and the International Council for Industrial and Applied Mathematics commissioned a joint study called the Educational Interfaces between Mathematics and Industry. During this study, I learned about the challenges colleagues around the world experienced while trying to train students to develop mathematical skills which they could use in industry. I was struck by how many institutions around the world had adopted variants of the successful IMA graduate student mathematicsin-industry training camp model, often explicitly crediting the format. The Canada-based Pacific Institute for the Mathematical Sciences now runs this event in collaboration with the IMA. The IMA industrial postdoctoral program was discussed as one of the more successful initiatives which institutions in other countries would like to adopt. The major hurdle we all identified-establishing links and long-term relationships with industrial partners-is one that the IMA has successfully surmounted.

As another example, the MBI runs a very successful summer graduate program to train young researchers in new topics at the interface of mathematics and the life sciences. These are in new topics, and most graduate programs do not offer courses in these. The format is nimble and effective, and MBI has accumulated a wealth of experience in running these programs well. Other countries would like to adopt similar initiatives and look to the MBI model. In 2012 the Center for Applied Mathematics in Biosciences and Medicine (in Canada) began to partner with MBI on this program. Many of us internationally recognize the importance of the life sciences in our times; we admire the innovative educational and research initiatives pioneered and supported at the MBI.

\section{A Final Word}

The institutes constitute major infrastructure in the mathematical sciences, akin to the large telescopes and particle accelerators in physics. What role do these institutes play in the research and training landscape? What is their broader impact? Do these roles need updating? What is being done well, and what needs to change? In my view, the community should have an opportunity for consultation on long-range plans and strategic investments in such infrastructure, especially if something with enormous impact is being phased out.

Both the IMA and MBI have a sustained track record of excellence in their respective missions and offer much-needed intellectual opportunities to a truly large number of mathematical scientists. They have a suite of programs which together generate a vibrant environment, much greater than the sum of the individual workshops. They have significantly contributed to a culture of change in our discipline, providing opportunities to researchers from diverse backgrounds and interests.

These institutes are important to a large number of mathematical scientists. Many were taken aback by the NSF recommendations and have sought venues to express their support of the IMA and the MBI. An open letter to the NSF indicating the importance of the IMA has been signed by over 3,400 mathematical scientists worldwide [5], including colleagues from universities, industry, and government labs. The signatories include SIAM Fellows and Fellows of the AMS. Many mathematical scientists at the beginning of their careers have also signed on; they recognize that the loss of these institutes will disproportionately impact them. This was a strong and constructive signal from members of the community about the critical importance of these resources.

The potential defunding of these institutes represents a large blow to the mathematical sciences at large and in particular to the development of novel applications of mathematics. I hope that the IMA and the MBI are able to continue their stellar work.

\section{References}

[1] www. noao.edu/currents/201110.htm] retrieved May 13, 2015.

[2] First Annual Report of the IMA, 1983. www. ima.umn. edu/ santosa/IMA1982report.pdf

[3] Report of the 2013 Committee of Visitors to the Division of Mathematical Sciences at the National Science Foundation, 2013, www.nsf.gov/mps/advisory/ covdocs/2013_DMS_COV_Report.pdf

[4] P. Holmes, R. J. Full, D. Koditsche and J. GuckenHEIMER, The dynamics of legged locomotion: models, analyses, and challenges, SIAM Rev. 48 (2006), no. 2, 207-304.

[5] https://sites.goog7e.com/site/friendsoftheima/ retrieved May 30, 2015. 